\title{
The Influence of Family Function on State Anxiety of Chinese College Students During the Epidemic of COVID-19
}

\author{
Lingping Yangt, Miao $\mathrm{Wu}^{+}$, Yuqi Wangt and Bin Peng* \\ College of Public Health and Management, Chongqing Medical University, Chongqing, China
}

OPEN ACCESS

Edited by:

Mike Z. Yao,

University of Illinois

at Urbana-Champaign, United States

Reviewed by:

Elena Commodari,

University of Catania, Italy

Angelica Moè

University of Padua, Italy

*Correspondence:

Bin Peng

pengbin@cqmu.edu.cn

tThese authors have contributed equally to this work and share first

authorship

Specialty section:

This article was submitted to

Health Psychology,

a section of the journal

Frontiers in Psychology

Received: 28 April 2021

Accepted: 28 June 2021

Published: 16 September 2021

Citation:

Yang L, Wu M, Wang Y and Peng B (2021) The Influence of Family Function on State Anxiety of Chinese College Students During the Epidemic

of COVID-19.

Front. Psychol. 12:701945. doi: 10.3389/fpsyg.2021.701945
The Coronavirus Disease 2019 (COVID-19) may affect mental health. There is little research about the influence of family function on the state anxiety of college students in the context of the global pandemic. The study aimed to clarify that generalized anxiety disorder (GAD) and trait anxiety had mediating effects in family function on the state anxiety of Chinese college students following the "stay-at-home" order during the outbreak of COVID-19. This cross-sectional study was conducted online with 1,039 respondents. We analyzed demographics, The State-Trait Anxiety Inventory (ST-Al) and Chinese Family Function Scale (FAD-18), Generalized Anxiety Disorder-7 (GAD-7), and used path analysis to discuss the influence of family function on state and trait anxiety. The results showed that female students' state and trait anxiety was higher than that of male students $(P<0.05)$. Medical students' state and trait anxiety was lower than that of literature students $(P<0.05)$. The GAD of the male was lower compared with the female. The score of family function has significant differences in gender, age, education, and region $(P<0.05)$. Gender, family function, state anxiety, trait anxiety, and GAD relate to others $(r=0.07 \sim 0.85, p<0.05)$. The results of fit indices for measurement invariance models showed that the impact of family function among GAD, state and trait anxiety across gender is significantly different (each step $p<0.05$ ). GAD and trait anxiety had a complete meditating effect between family function and state anxiety (the proportion of standard indirect mediating effect was $24.94 \%$ in females and $36.79 \%$ in males). A healthy family function may alleviate GAD and anxiety of college students during the COVID-19 pandemic.

Keywords: COVID-19, Chinese college student, anxiety, family function, mediating effect analysis

\section{INTRODUCTION}

Coronavirus disease 2019 (COVID-19) is an acute respiratory infectious disease caused by the 2019 Coronavirus. As we all know, COVID-19 is such a dreadful disease that has been "raging" on earth from November 2019 up to now (Liu Q. et al., 2020). Thus, many countries have adopted various methods to prevent more people from being infected. The Chinese government has taken many practical measures in the early stage, such as "lockdown" of the cities, and the order to "stay at home" (Wang M. W. et al., 2020). Brooks et al. (2020) reported that quarantine could bring some 
psychological diseases such as post-traumatic stress symptoms, confusion, and anger. Turkmen et al. (2020) said that lengthy quarantine could increase psychosocial stress and lead to "stress-sensitive" diseases. These studies showed that lengthyhome quarantine might cause ill mental health in the social environment of the epidemic.

The first cause of disability is mental health problems, a significant public health issue worldwide (Ramon-Arbues et al., 2020). In particular, the prevalence of depression and anxiety in developing countries is $10-44 \%$. What is worse, it is the fourth leading cause of morbidity (Azad et al., 2017). Awadalla et al. (2020) noted the marked rates of depression and anxiety in student populations and the obvious potential negative implications for academic study. They can have a negative impact on engagement with their course of study. Students experiencing depression may miss more classes, tests, and assignments, and are more likely to drop courses than their non-depressed peers (Pedrelli et al., 2015). Moreover, it is reported that anxiety has significant adverse effects on academic success, achievement, well-being, and so on (Haller et al., 2015). However, the proportion of depression and anxiety symptoms in college students is very high (RamonArbues et al., 2020). Full-time college students had more time with family members during the "stay-at-home" time. Some evidence showed that family function significantly influences family members' physiology, psychology, and sociality (Ahlberg et al., 2020). Gonzálvez et al. (2019) showed that good family function would reduce students' anxiety. However, there are few studies on the impact of such a situation on college students.

Thus, researchers believed that family function might have a significant impact on the generalized anxiety disorder (GAD), state, and trait anxiety of Chinese college students who have to stay at home during the epidemic of COVID-19. Therefore, path analysis was used in the structural equation model (SEM) to discover the relationship of family function, GAD, state anxiety, and trait anxiety.

\section{Study Aim and Hypotheses}

Based on these considerations, the main aim of this study was to investigate the impact of family function on state anxiety by GAD and trait anxiety during the pandemic in Chinese college students. In particular, the purpose of the study was to analyze sociodemographic characterization, the status of family function, state anxiety, trait anxiety and GAD in Chinese college students, the relationship between family function, state anxiety, trait anxiety, and GAD. Moreover, the study explored other sociodemographic variables influence of family function on state anxiety by GAD and trait anxiety. More specifically, the study intended to verify the following hypotheses:

Hypothesis $1(\mathrm{H} 1)$ : Females are more anxious than males. Hypothesis 2 (H2): GAD and trait anxiety mediate family function and state anxiety.

Hypothesis 3 (H3): Gender and major moderated the relationship between family function and state anxiety.

\section{MATERIALS AND METHODS}

\section{Setting and Participants}

This study is a cross-sectional study to survey the influence of family function on state and trait anxiety of Chinese college students during the COVID-19 epidemic. We have to choose an online survey because of the government's requirements to stay at home. The questionnaire was distributed via Wenjuanxing, ${ }^{1}$ which is an online survey platform; on WeChat (Tencent, Shenzhen, China) and other social platforms (QQ, Tencent, Shenzhen, China). The questionnaire was distributed from March 14 to 21,2020 . The questionnaire link was first sent to the students' class group and collected by snowball sampling. Then the first respondent forwarded the questionnaire to others.

Specifically, based on the following criteria: age $>15$, college students, filling time $>10 \mathrm{~min}$, respondents without a history of anxiety disorders. The same Internet protocol address, WeChat ID or QQ number were excluded to avoid multiple compilations. The questionnaire with obvious logical errors was eliminated.

\section{Variables}

\section{Sociodemographic Characterization}

In the sociodemographic survey, respondents were invited to answer simple questions regarding gender, age, education, region, and major. Then they were asked to answer questions related to COVID-19. For example, whom you live with during the epidemic, whether someone around you is infected with COVID-19, the frequency of going out, and the longest time spent at home.

\section{Anxiety}

State-Trait Anxiety Inventory (ST-AI; Spielberger, 1970) is suitable for evaluating the state and trait anxiety of Chinese College Students. It comprises two blocks (Form Y1 and Form Y2) of 20 items, evaluated in a four-point Likert scale. Form Y1 is used to evaluate transient or temporary anxiety (state anxiety). Form Y2 is used to assesse dispositional or general anxiety (trait anxiety). The score is generated by the sum of the 20 items for each scale. The respondents with a higher score might have a higher anxiety degree. Internal consistency in this study proved to be good (state $\alpha=0.93$; trait $\alpha=0.90$ ).

\section{GAD}

Generalized Anxiety Disorder-7 (GAD-7) is also suitable for evaluating the GAD of Chinese college students. GAD-7 (Spitzer et al., 2006) consists of seven items measuring worry and anxiety symptoms. Each item is scored on a four-point Likert scale (0-3) with total scores ranging from 0 to 21 , with higher scores reflecting greater anxiety severity. Scores above ten are considered to be in the clinical range (Spitzer et al., 2006). The GAD-7 has shown good reliability and construct validity (Kroenke et al., 2007; Lowe et al., 2008).

\footnotetext{
${ }^{1}$ www.wjx.cn
} 


\section{Family Function}

FAD-18 (Manyan Zhang, 2019) can effectively evaluate the family function of Chinese college students. There are 18 items on the scale, including intimacy, adaptability, control, and growth of four dimensions. Each item is scored on a scale of $0-1$ with total scores ranging from 0 to 18 , with higher scores reflecting worse family function. Internal consistency in this study proved to be good (total $\alpha=0.87$, four dimensions $\alpha=0.82,0.76,0.73$, and 0.59 , respectively).

\section{Statistical Analysis}

Descriptive and correlation analysis of collected data was analyzed by SPSS23.0 Version (IBM SPSS Statistics, New York, NY, United States). Path analysis in SEM used AMOS 24.0. Quantitative data were analyzed by ANOVA (analysis of variance). Correlation analysis was used to analyze the relationship between factors. The results of the STAI scale were also compared with the healthy norm (Spielberger, 1970) by $Z$-test. Measurement invariance was used to analyze group differences across gender. The mediating effect analysis was used to discover the relationship among family function, GAD, state and trait anxiety. Bootstrapping was used to analyze the mediating effect. Samples were randomly selected 10,000 times. $P<0.05$ was considered statistically significant.

\section{RESULTS}

\section{Respondents' Demographic Characteristics}

One thousand two hundred fifty nine questionnaires were submitted. We had eliminated the questionnaires that did not meet the requirements and 1,200 of them were valid. Among the 1,200 questionnaires, 161 questionnaires were excluded due to errors. Finally, there were 1,039 questionnaires left. These respondents were from 30 provinces and province-level municipalities. They were distributed in different majors, grades and living environments. Thus, we are convinced that these respondents can represent Chinese college students.

The results showed that males and females had a significant difference in state anxiety score $(p<0.01)$ and trait anxiety score $(p<0.01)$. It could be easily found that males' state and trait anxiety scores were lower than females'. What is more, state anxiety and trait anxiety scores were significantly different between medical respondents and other majors $(p<0.01)$. The scores of state and trait anxiety in other majors were higher than those in medicine. Other factors had no difference.

The family function had a significant difference in gender, age, region, and education. That of the male was better than that of the female $(p<0.05)$. What is more, the older students had a better family function $(p<0.01)$. Those respondents with a high degree of education had a better family function $(p<0.05)$. Significantly, the family function was better in the region where the epidemic was not serious $(p<0.05)$. The score of GAD in gender was different. Similarly, the male had lower scores than the female $(p<0.05)$. Detailed results could be found in Table 1 .
There was no significant difference between the respondents and the healthy norm excluded the score of trait anxiety in females. In this survey, the scores of state and trait anxiety of females were $38.36 \pm 8.91$ and $39.45 \pm 8.90$ accordingly, and those of males were $36.84 \pm 10.26$ and $38.04 \pm 9.86$. Similar to the healthy norm, the scores of females were higher than those of males in both state and trait anxiety, and the trait anxiety scores of both females and males were higher than state anxiety. Females had a lower score of trait anxiety than the norm $(p<0.05)$. Detailed results could be found in Table 2 .

\section{Multivariate ANOVA}

The results showed that interaction between gender and major had no statistical significance $(p>0.05)$. Only gender showed significant difference in state anxiety, trait anxiety, family function, and GAD. Detailed results could be found in Table 3.

\section{Correlation Analysis}

There were positive correlations among gender, family function, state anxiety, trait anxiety and GAD. Correlation coefficient $r=0.07 \sim 0.85(p<0.05)$. A detailed description of the results could be found in Table 4 .

\section{Path Analysis}

Because it was a saturated model, which means that the number of estimated parameters exactly equal to the elements in the covariance matrix (the degree of freedom is 0 ), researchers no longer estimated its fit indices; but only focus on its path coefficients. Considering family function as an independent variable, state anxiety as a dependent variable and GAD and trait anxiety as mediating variables across gender, the results of bootstrapping showed that GAD and trait anxiety had complete mediating effects between family function and state anxiety. Gender invariance testing yielded similar models for female and male respondents, with significant chi-square for any step difference $(p<0.05)$. Detailed fit indices for measurement invariance models across gender could be found in Table 5 .

The proportion of standard indirect mediating effect was $24.94 \%$ in females and $36.79 \%$ in males. The estimation of standard direct effect was -0.023 in females $(p=0.306)$ and -0.040 in males $(p=0.263)$. Detailed bootstrapping results of estimated path coefficients could be found in Figure 1 and Table 6.

\section{DISCUSSION}

The vast majority of respondents had never been outside. It showed the fact that prophylactic measures implemented by China were effective. Moreover, it was not hard to find that most of the respondents lived with their parents. The study of Gonzálvez et al. (2019) may explain that the respondents with better family function have lower anxiety levels. The results in Table 2 showed no significant difference between respondents, and the norm excluded the trait anxiety of females. The result of Liu X. et al. (2020) was the same as ours. Due to implementing the "stay-at-home" order, college students may be exposed to 
TABLE 1 | The demographic characteristics and ANOVA results of the respondents.

\begin{tabular}{cccccccc}
\hline Variable & Number & Proportion & $F$ value & $\begin{array}{c}\text { State } \\
\text { anxiety }\end{array}$ & $\begin{array}{c}\text { Trait } \\
\text { anxiety }\end{array}$ & $\begin{array}{c}\text { Family } \\
\text { function }\end{array}$ \\
\cline { 2 - 6 } & $\begin{array}{l}\text { (Total, } \\
n=1039)\end{array}$ & $(\%)$ & $P$-value & Mean \pm SD & Mean \pm SD & Mean \pm SD & Mean \pm SD \\
& & & & & &
\end{tabular}

\section{Gender}

$\begin{array}{lll}\text { Male } & 344 & 33.11 \\ & 695 & 66.89\end{array}$

Female

695

Age (years)

$\begin{array}{lll}\leq 22 & 547 & 52.65 \\ >22 & 492 & 47.35\end{array}$

Region

\begin{tabular}{lcc}
$\begin{array}{l}\text { Severe } \\
\text { epidemic } \\
\text { city }\end{array}$ & 109 & 10.49 \\
$\begin{array}{l}\text { Moderate } \\
\text { epidemic }\end{array}$ & 63.91 \\
city & \\
$\begin{array}{l}\text { Mild } \\
\text { epidemic }\end{array}$ & \\
city & 266 & 25.6 \\
\hline
\end{tabular}

Major

$\begin{array}{lll}\text { Medicine } & 594 & 57.17 \\ \text { Science } & 296 & 28.49 \\ \text { and } & \\ \text { Engineering } & \\ \text { Literature } & 149 & 14.34\end{array}$

Education

$\begin{array}{lll}\text { Undergraduate } & 650 & 62.56 \\ \text { Postgraduate } & 389 & 37.44\end{array}$

Number of people living together during the epidemic

$\begin{array}{lll}\leq 4 & 767 & 73.82 \\ >4 & 272 & 26.18\end{array}$

Live with

$\begin{array}{lcc}\begin{array}{l}\text { Without } \\ \text { parents }\end{array} & 120 & 11.55 \\ \begin{array}{l}\text { Single } \\ \text { parent }\end{array} & 111 & 10.68 \\ \text { Parents } & & \\ \text { Parents and } & 181 & 60.35 \\ \text { grandparents } & & 17.42\end{array}$

$\begin{array}{cccc}36.84 \pm 10.26 & 38.04 \pm 9.86 & 4.09 \pm 2.78 & 3.87 \pm 3.66 \\ 38.36 \pm 8.91 & 39.45 \pm 8.90 & 4.67 \pm 3.39 & 4.38 \pm 3.36 \\ 6.00 & 5.37 & 7.56 & 5.00 \\ 0.01 & 0.02 & 0.01 & 0.03\end{array}$

$F$ value

$P$ value

$38.09 \pm 9.17$
$37.60 \pm 9.66$
0.71
0.40

$38.49 \pm 10.18$

$37.77 \pm 9.28$

$37.82 \pm 9.42$

$F$ value

$P$ value

$$
\begin{aligned}
& 0.28 \\
& 0.76
\end{aligned}
$$

$37.48 \pm 9.13$

$37.66 \pm 9.75$
$39.74 \pm 9.61$

$$
5.21
$$$$
0.02
$$

$F$ value

$P$ value

$F$ value
$P$ value

$F$ value

$P$ value

F value
$P$ value
$38.14 \pm 9.14$
$37.50 \pm 9.71$
1.21
0.27

$37.85 \pm 9.52$
$37.87 \pm 9.09$
0.00
0.98

$38.91 \pm 9.91$

$38.41 \pm 8.63$

$37.68 \pm 9.41$

$37.42 \pm 9.49$

$39.30 \pm 9.09$
$38.64 \pm 9.41$
1.34
0.25

$4.80 \pm 3.47$

$4.13 \pm 2.87$

11.35

$<0.01$
$4.23 \pm 3.45$
$4.18 \pm 3.50$
0.05
0.82

$39.97 \pm 10.57$

$4.96 \pm 3.28$

$4.11 \pm 3.08$

$38.87 \pm 9.09$

$4.57 \pm 3.24$

$4.20 \pm 3.51$

$38.87 \pm 9.06$

$4.02 \pm 3.06$

$4.49 \pm 4.05$

0.69

0.50

3.98

0.02

0.44

0.65

$38.39 \pm 9.06$

$4.60 \pm 3.24$

$4.11 \pm 3.46$

$39.28 \pm 9.58$

$4.24 \pm 2.90$

$4.16 \pm 3.38$
$40.78 \pm 9.10$
8.27
0.00
$39.40 \pm 8.96$
$38.48 \pm 9.58$
2.54
0.11
$38.94 \pm 9.37$
$39.11 \pm 8.91$
0.06
0.80

$38.89 \pm 9.37$

$38.73 \pm 9.27$

0.38

0.77
$4.46 \pm 3.65$
1.02
0.31
$4.70 \pm 3.69$
2.57
0.11

$\begin{array}{cc}4.72 \pm 3.40 & 4.20 \pm 3.45 \\ 4.18 \pm 2.94 & 4.22 \pm 3.50 \\ 7.28 & 0.01 \\ 0.01 & 0.92\end{array}$

$\begin{array}{cc}4.53 \pm 3.29 & 4.16 \pm 3.52 \\ 4.33 \pm 2.98 & 4.34 \pm 3.32 \\ 0.74 & 0.53 \\ 0.39 & 0.47\end{array}$

$39.79 \pm 9.45$

$4.46 \pm 3.45$

$4.22 \pm 3.89$

$4.65 \pm 3.31$

$4.31 \pm 3.41$

$4.49 \pm 3.23$

$4.18 \pm 3.42$

$4.35 \pm 2.94 \quad 4.25 \pm 3.44$

$\begin{array}{llll}0.83 & 0.38 & 0.19 & 0.05 \\ 0.48 & 0.77 & 0.89 & 0.99\end{array}$

(Continued) 
TABLE 1 | Continued

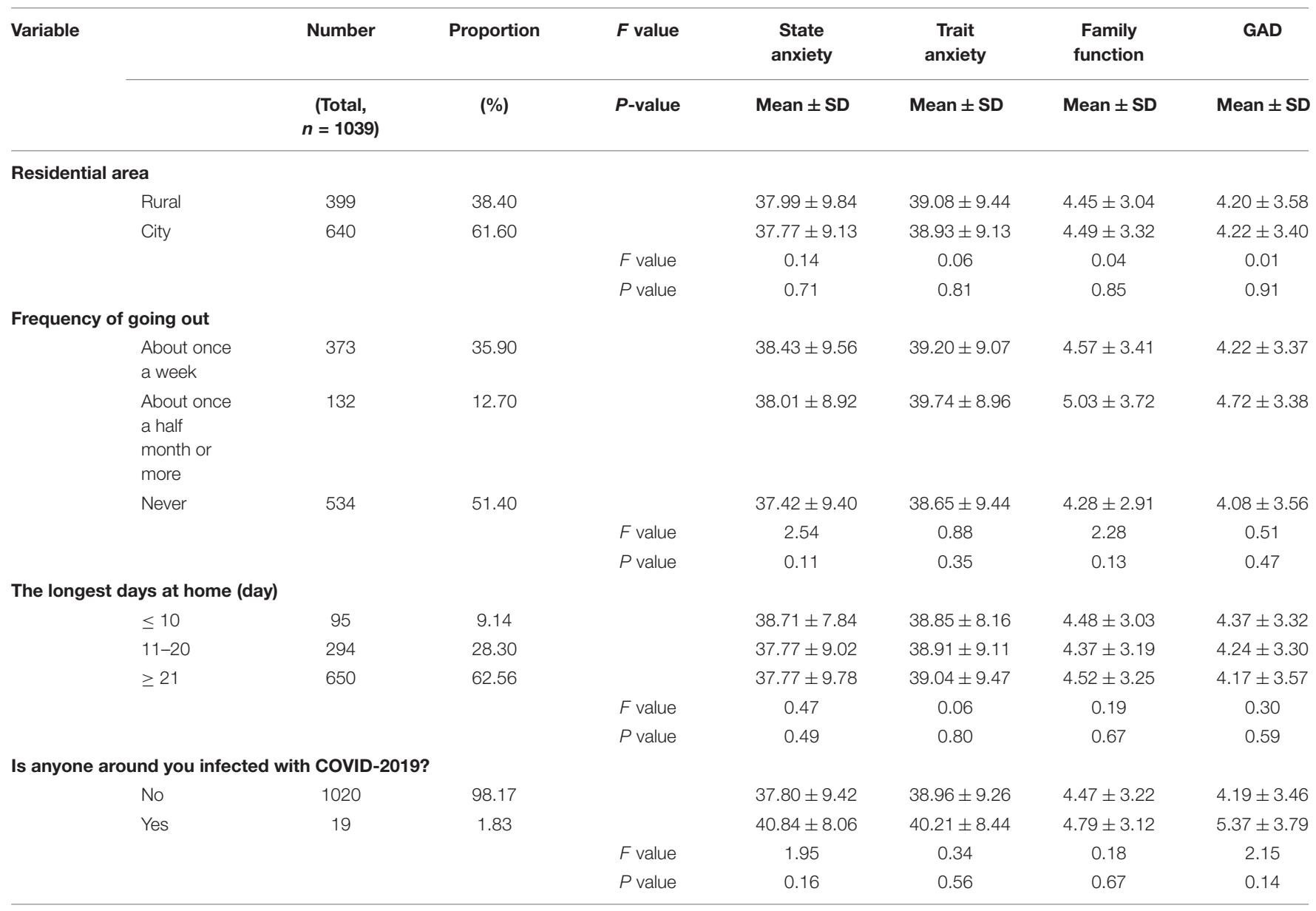

TABLE 2 | The Z-test analysis results of state and trait anxiety compared with norm.

\begin{tabular}{|c|c|c|c|c|}
\hline \multirow[t]{2}{*}{ Anxiety } & \multirow[t]{2}{*}{ Gender } & \multicolumn{2}{|c|}{ Scores } & \multirow[t]{2}{*}{$P$-value } \\
\hline & & Norm & Samples & \\
\hline State anxiety & Female & $38.76 \pm 11.95$ & $38.36 \pm 8.91$ & 0.38 \\
\hline \multirow[t]{2}{*}{ Trait anxiety } & Male & $38.30 \pm 9.18$ & $38.04 \pm 9.86$ & 0.60 \\
\hline & Female & $40.40 \pm 10.15$ & $39.45 \pm 8.90$ & $<0.05$ \\
\hline
\end{tabular}

TABLE 3 | The results of Multivariate ANOVA.

\begin{tabular}{|c|c|c|c|c|c|c|c|c|}
\hline \multirow[t]{2}{*}{ Variable } & \multicolumn{2}{|c|}{ State anxiety } & \multicolumn{2}{|c|}{ Trait anxiety } & \multicolumn{2}{|c|}{ Family function } & \multicolumn{2}{|c|}{ GAD } \\
\hline & $F$ value & $P$-value & $F$ value & $P$-value & $F$ value & $P$-value & $F$ value & $P$-value \\
\hline Major & 1.63 & 0.16 & 2.30 & 0.06 & 0.59 & 0.67 & 1.89 & 0.11 \\
\hline Gender*Major & 1.44 & 0.22 & 1.14 & 0.33 & 1.54 & 0.19 & 0.97 & 0.42 \\
\hline
\end{tabular}

${ }^{*} P<0.05$ and ${ }^{* *} P<0.01$.

social media for a longer time, thus receiving more information about COVID-19. Some studies (Li et al., 2020) showed that people might produce vicarious traumatization (VT) in the case of such social events. It is a kind of psychological abnormality indirectly caused by witnessing many cruel and destructive scenes, and the degree of damage exceeds the psychological and emotional tolerance limit of some people (Molnar et al., 2020). However, considering that it has been nearly 4 months since 
TABLE 4 | The results of Pearson correlation analysis among gender, state anxiety, trait anxiety, family function, and GAD.

\begin{tabular}{|c|c|c|c|c|}
\hline Variable & Trait anxiety & Family function & GAD & Gender $^{a}$ \\
\hline Trait anxiety & & $0.32^{\star \star \star}$ & $0.67^{\star \star \star}$ & $0.07^{\star}$ \\
\hline GAD & & & & $0.07^{\star}$ \\
\hline
\end{tabular}

${ }^{*} P<0.05,{ }^{* *} P<0.01$, and ${ }^{* * *} P<0.001$.

${ }^{a}$ Biserial correlation coefficient.

TABLE 5 | Fit indices for measurement invariance models for men and women: baseline (unconstrained), weak (measurement weights), strong (measurement intercept), and strict (measurement residual) $(N=1039)$.

\begin{tabular}{lcccc}
\hline Parameter & Baseline & Weak & Strong & Strict \\
\hline $\mathrm{Chi}^{2}(\mathrm{df})$ & $0.000(0)$ & $17.639(6)$ & $35.070(7)$ & $47.431(1)$ \\
$\Delta \mathrm{Chi}^{2}(\Delta \mathrm{df})$ & - & $17.639(6)$ & $0.987^{\star \star *}$ & $10.889(10)$ \\
$\mathrm{CFI}$ & 1.000 & $0.995^{\star *}$ & 0.062 & $0.983^{\star}$ \\
RMSEA & - & 0.043 & 0.059 \\
\hline
\end{tabular}

${ }^{*} P<0.05,{ }^{* *} P<0.01$, and ${ }^{* * *} P<0.001$.

CFI, Comparative fit index; RMSEA, Root-mean-square error of approximation.
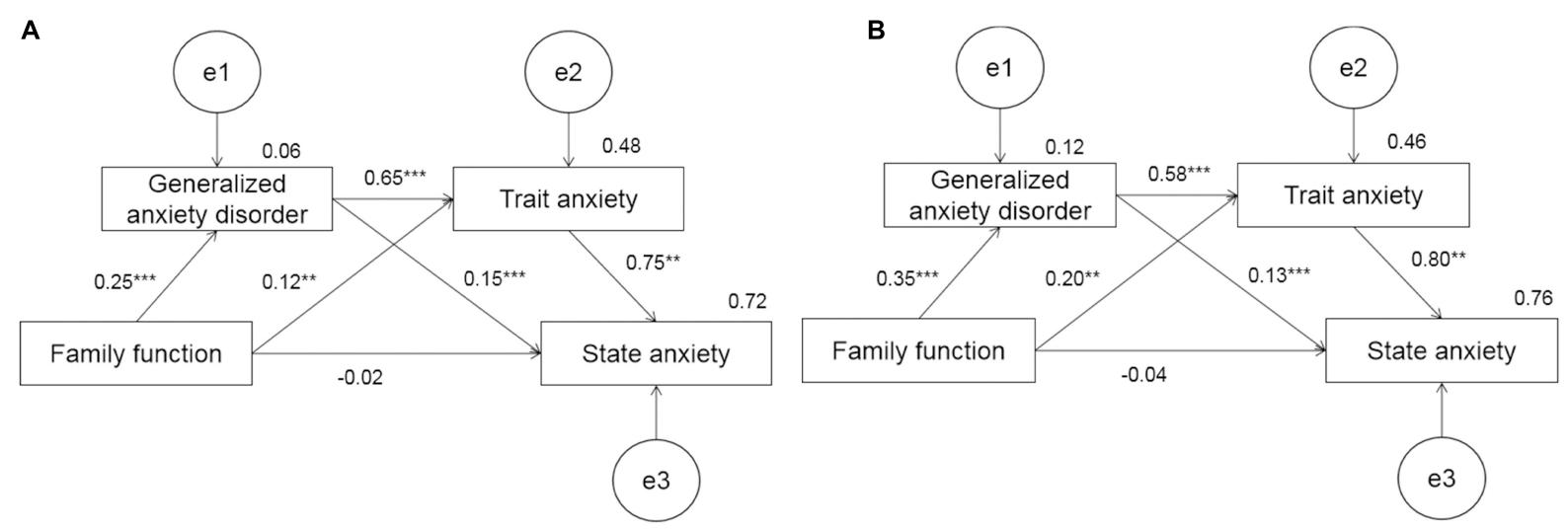

FIGURE 1 | Standard path analysis of family function, GAD, state, and trait anxiety for females and males. (A) Female and (B) male are included. ${ }^{* *} P<0.01$ and ${ }^{\star \star \star *} P<0.001$.

TABLE 6 | The results of estimation about standard parameters in path coefficients among family function, GAD, state anxiety, and trait anxiety for females and males.

\begin{tabular}{lcc}
\hline & & Coefficients $(\mathbf{9 5 \%} \mathbf{C l})$ \\
\cline { 2 - 3 } Path & Female & Male \\
\hline GAD $\leftarrow$ Family function & $0.246(0.171,0.320)^{\star \star \star}$ & $0.347(0.229,0.450)^{\star \star \star}$ \\
Trait anxiety GAD & $0.654(0.605,0.700)^{\star \star \star}$ & $0.584(0.506,0.651)^{\star \star \star}$ \\
Trait anxiety $\leftarrow$ Family function & $0.119(0.057,0.179)^{\star \star}$ & $0.196(0.117,0.271)^{\star \star \star}$ \\
State anxiety $\leftarrow$ GAD & $0.150(0.092,0.207)^{\star \star \star}$ & $0.135(0.055,0.213)^{\star \star}$ \\
State anxiety $\leftarrow$ Family function & $-0.023(-0.066,0.021)$ & $-0.040(-0.112,0.030)$ \\
State anxiety $\leftarrow$ Trait anxiety & $0.746(0.699,0.7994)^{\star \star \star}$ & $0.796(0.716,0.869)^{\star \star \star}$ \\
\hline
\end{tabular}

${ }^{* *} P<0.01$ and ${ }^{* * *} P<0.001$.

the outbreak of the COVID-19 to this survey, the respondents may have developed compassion fatigue (Hayuni et al., 2019), a psychological self-protection mechanism of the human body. Elena Commodari et al. (Commodari and La Rosa, 2020) reported that Italian adolescents had a low perception of risk of
COVID-19, perceived comparative susceptibility and perceived seriousness because of high trust in their good health. What is more, it is reported that good support for prevention and control policies is negatively associated with depression among respondents (Ding et al., 2020). China controlled the epidemic 
well in a short time. Therefore, that may be one reason why there was little difference between the respondents and the healthy norm.

The score of scales in males was lower than that in females $(p<0.05)$. This may indicate that females may become more anxious and vulnerable to social events, which was similar to the results of Zhong et al. (Bäuerle et al., 2020; Wang C. et al., 2020; Zhong et al., 2020). The state and trait anxiety scores of medical college students are lower than those of literature. Studies showed that (Chang et al., 2020) medical respondents shave excellent professional foundation and a better understanding of COVID-19, while literature respondents suffered more anxiety. A family function is related to gender, age, education, and region. Mainly, the family function performed better in the less severe epidemic region. Therefore, it indicated that family function may be related to COVID-19.

The research of Leslie et al. (2015) and Moe et al. (2021) said that females' underrepresentation has been linked to gender stereotypes and ability-related beliefs as well as gender differences in specific cognitive abilities. Ceci et al. (2014) reported that females in different majors might be affected by family factors more than males. But our results showed that there is no interaction of gender and major in state, trait anxiety, family function and $\operatorname{GAD}(p>0.05)$ and major had no difference in multivariate ANOVA analysis. It may be indicated that gender affected family function on anxiety while major was not.

Although other factors were not significant, we also found some notable points. The results showed that with parents around, less anxiety was experienced. The average scores of state anxiety in respondents who went out frequently were highest. This suggested that those respondents may still be anxious about COVID-19. Our results were similar to those of others (Elrashidi et al., 2018; Ayhan Başer et al., 2020; French et al., 2020; Jacobson et al., 2020; Temsah et al., 2020; Tull et al., 2020).

Generalized anxiety disorder and trait anxiety had complete mediating effects between family function and state anxiety, which indicates that family function has no direct effect on state anxiety in the short term during the epidemic period. It can be significantly influenced by trait anxiety and GAD. However, the research of Shain et al. (2020) said that, in general, state anxiety could be directly influenced by family function. It indicated that COVID-19 might have a more significant impact on family function during "stay-at-home" time. Angelica Moè et al. (Moè and Putwain, 2020) reported that females might be more easily affected by external cause than males. We could quickly find the results from measurement invariance that family function on state anxiety differed across gender. It may be suggested that during the outbreak of COVID-19, the impact of family function on trait anxiety of females was less than that of males, which suggested that females were vulnerable to social events (Kelly, 2020).

\section{Practical Implications}

The theoretical implication of this study is the analysis of how family function affects state anxiety by GAD and trait anxiety across gender. In particular, trait anxiety and GAD had a positive impact on state anxiety. Besides, GAD and trait anxiety are mediating effects between family function and state anxiety. Thus, this justifies the model of this study, which links family function with state anxiety. Moreover, this may offer much evidence for further study.

This study also has social implications. As an essential part of society, a family affected many aspects of family members. Especially, college students dependent on family more than others. Health education can increase the family function among the public. In addition, a healthy family function can alleviate the anxiety and depression of college students.

\section{Limitations}

This study also had limitations. Firstly, the collection of data was conducted by snowball resampling not based on representative sampling. Secondly, there was a significant difference in the number of people in some groups in this study, which may impact the analysis results. Thirdly, some factors had not been observed and not been included in the study, which will affect the final results. Last, among the respondents in our study, few of them had COVID-19 cases around them. Therefore, the study's conclusion may only apply to Chinese college students who do not have COVID-19 cases around them.

\section{CONCLUSION}

Some significant factors related to this study were also found. Among Chinese college students, females were more likely to be affected by social events, and aggravating their degree of anxiety. Furthermore, we also found that medical college students were less anxious about pandemic infectious diseases than literature students. Those who had a better family function had less anxiety. The family function had a significant impact on trait anxiety and GAD, and indirectly impacted upon state anxiety. Thus, the mental health of females should be paid more attention to. A better family function can alleviate the anxiety of college students during the epidemic of COVID-19. Generally, the significance of this study is profound.

\section{DATA AVAILABILITY STATEMENT}

The raw data supporting the conclusion of this article will be made available by the authors, without undue reservation.

\section{ETHICS STATEMENT}

The studies involving human participants were reviewed and approved by the Ethics Committee of Chongqing Medical University. Written informed consent from the participants' 
legal guardian/next of kin was not required to participate in this study in accordance with the national legislation and the institutional requirements.

\section{AUTHOR CONTRIBUTIONS}

BP and LY conceived the presented idea and performed the data analysis. LY, MW, and YW performed material preparation and assisted with the recruitment process and distribution

\section{REFERENCES}

Ahlberg, M., Hollman Frisman, G., Bertero, C., and Agren, S. (2020). Family health conversations create awareness of family functioning. Nurs. Crit. Care 25, 102-108. doi: 10.1111/nicc. 12454

Awadalla, S., Davies, E. B., and Glazebrook, C. (2020). A longitudinal cohort study to explore the relationship between depression, anxiety and academic performance among emirati university students. BMC Psychiatry 20:448. doi: 10.1186/s12888-020-02854-z

Ayhan Başer, D., Çevik, M., Gümüştakim, Ş, and Başara, E. (2020). Assessment of individuals' attitude, knowledge and anxiety towards Covid-19 at the first period of the outbreak in Turkey: a web-based cross-sectional survey. Int. J. Clin. Pract. 74:e13622. doi: 10.1111/ijcp.13622

Azad, N., Shahid, A., Abbas, N., Shaheen, A., and Munir, N. (2017). Anxiety and depression in medical students of a private medical college. J. Ayub. Med. Coll Abbottabad. 29, 123-127.

Bäuerle, A., Teufel, M., Musche, V., Weismüller, B., Kohler, H., Hetkamp, M., et al. (2020). Increased generalized anxiety, depression and distress during the covid19 pandemic: a cross-sectional study in germany. J. Public Health 42, 672-678. doi: 10.1093/pubmed/fdaa106

Brooks, S. K., Webster, R. K., Smith, L. E., Woodland, L., Wessely, S., Greenberg, N., et al. (2020). The psychological impact of quarantine and how to reduce it: rapid review of the evidence. Lancet 395, 912-920. doi: 10.1016/S0140-6736(20) 30460-8

Ceci, S. J., Ginther, D. K., Kahn, S., and Williams, W. M. (2014). Women in academic science: a changing landscape. Psychol. Sci. Public Int. 15, 75-141. doi: $10.1177 / 1529100614541236$

Chang, J., Yuan, Y., and Wang, D. (2020). [mental health status and its influencing factors among college students during the epidemic of covid-19]. Nan Fang Yi Ke Da Xue Xue Bao 40, 171-176. doi: 10.12122/j.issn.1673-4254.2020. 02.06

Commodari, E., and La Rosa, V. L. (2020). Adolescents in quarantine during covid19 pandemic in italy: perceived health risk, beliefs, psychological experiences and expectations for the future. Front. Psychol. 11:559951. doi: 10.3389/fpsyg. 2020.559951

Ding, Y., Xu, J., Huang, S., Li, P., Lu, C., and Xie, S. (2020). Risk perception and depression in public health crises: evidence from the covid-19 crisis in china. Int. J. Environ. Res. Public Health 17:5728. doi: 10.3390/ijerph1716 5728

Elrashidi, M. Y., Philpot, L. M., Ramar, P., Leasure, W. B., and Ebbert, J. O. (2018). Depression and anxiety among patients on chronic opioid therapy. Health Serv. Res. Manag. Epidemiol. 5:2333392818771243. doi: 10.1177/2333392818771243

French, M. T., Mortensen, K., and Timming, A. R. (2020). Psychological distress and coronavirus fears during the initial phase of the covid-19 pandemic in the united states. J. Ment. Health Policy Econ. 23, 93-100.

Gonzálvez, C., Díaz-Herrero, Á, Sanmartín, R., Vicent, M., Pérez-Sánchez, A. M., and García-Fernández, J. M. (2019). Identifying risk profiles of school refusal behavior: differences in social anxiety and family functioning among spanish adolescents. Int. J. Environ. Res. Public Health 16:3731. doi: 10.3390/ ijerph16193731

Haller, S. P., Cohen Kadosh, K., Scerif, G., and Lau, J. Y. (2015). Social anxiety disorder in adolescence: how developmental cognitive neuroscience findings may shape understanding and interventions for psychopathology. Dev. Cogn. Neurosci. 13, 11-20. doi: 10.1016/j.dcn.2015.02.002 of the surveys. LY performed data management. All authors contributed to the study's conception and design, writing and review of the manuscript, and read and approved the final manuscript.

\section{FUNDING}

This work was funded by the National Key Research and Development Program of China (2018YFC1311700).

Hayuni, G., Hasson-Ohayon, I., Goldzweig, G., Bar Sela, G., and Braun, M. (2019). Between empathy and grief: the mediating effect of compassion fatigue among oncologists. Psychooncology 28, 2344-2350. doi: 10.1002/pon.5227

Jacobson, N. C., Lekkas, D., Price, G., Heinz, M. V., Song, M., O’Malley, A. J., et al. (2020). Flattening the mental health curve: Covid-19 stay-at-home orders are associated with alterations in mental health search behavior in the united states. JMIR Ment. Health 7:e19347. doi: 10.2196/19347

Kelly, B. D. (2020). Coronavirus disease: challenges for psychiatry. Br. J. Psychiatry 217, 352-353. doi: 10.1192/bjp.2020.86

Kroenke, K., Spitzer, R. L., Williams, J. B., Monahan, P. O., and Lowe, B. (2007). Anxiety disorders in primary care: prevalence, impairment, comorbidity, and detection. Ann. Int. Med. 146, 317-325. doi: 10.7326/0003-4819-146-5200703060-00004

Leslie, S. J., Cimpian, A., Meyer, M., and Freeland, E. (2015). Expectations of brilliance underlie gender distributions across academic disciplines. Science 347, 262-265. doi: 10.1126/science. 1261375

Li, Z., Ge, J., Yang, M., Feng, J., Qiao, M., Jiang, R., et al. (2020). Vicarious traumatization in the general public, members, and non-members of medical teams aiding in covid-19 control. Brain Behav. Immun. 88, 916-919. doi: 10. 1016/j.bbi.2020.03.007

Liu, Q., Xu, K., Wang, X., and Wang, W. (2020). From sars to covid-19: what lessons have we learned? J. Infect. Public Health 13, 1611-1618. doi: 10.1016/ j.jiph.2020.08.001

Liu, X., Luo, W. T., Li, Y., Li, C. N., Hong, Z. S., Chen, H. L., et al. (2020). Psychological status and behavior changes of the public during the covid-19 epidemic in china. Infect. Dis. Poverty 9:58. doi: 10.1186/s40249-020-00678-3

Lowe, B., Decker, O., Muller, S., Brahler, E., Schellberg, D., Herzog, W., et al. (2008). Validation and standardization of the generalized anxiety disorder screener (gad-7) in the general population. Med. Care 46, 266-274. doi: 10.1097/MLR. 0b013e318160d093

Manyan Zhang, Y. Y. (2019). Preparation of the chinese family function scale and reliability and validity study. Psychol. Commun. 2, 101-108.

Moe, A., Hausmann, M., and Hirnstein, M. (2021). Gender stereotypes and incremental beliefs in stem and non-stem students in three countries: relationships with performance in cognitive tasks. Psychol. Res. 85, 554-567. doi: 10.1007/s00426-019-01285-0

Moè, A., and Putwain, D. W. (2020). An evaluative message fosters mathematics performance in male students but decreases intrinsic motivation in female students. Educ. Psychol. 40, 941-960. doi: 10.1080/01443410.2020.1730767

Molnar, B. E., Meeker, S. A., Manners, K., Tieszen, L., Kalergis, K., Fine, J. E., et al. (2020). Vicarious traumatization among child welfare and child protection professionals: a systematic review. Child Abuse Negl. 110(Pt 3):104679. doi: 10.1016/j.chiabu.2020.104679

Pedrelli, P., Nyer, M., Yeung, A., Zulauf, C., and Wilens, T. (2015). College students: mental health problems and treatment considerations. Acad. Psychiatry 39, 503-511. doi: 10.1007/s40596-014-0205-9

Ramon-Arbues, E., Gea-Caballero, V., Granada-Lopez, J. M., Juarez-Vela, R., Pellicer-Garcia, B., and Anton-Solanas, I. (2020). The prevalence of depression, anxiety and stress and their associated factors in college students. Int. J. Environ. Res. Public Health 17:7001. doi: 10.3390/ijerph17197001

Shain, L. M., Pao, M., Tipton, M. V., Bedoya, S. Z., Kang, S. J., Horowitz, L. M., et al. (2020). Comparing parent and child self-report measures of the state-trait anxiety inventory in children and adolescents with a chronic health condition. J. Clin. Psychol. Med. Settings 27, 173-181. doi: 10.1007/s10880-019-09631-5 
Spielberger, C. D. (1970). Manual for the State-Trait Anxiety Inventory. ("Self Evaluation Questionnaire"). Palo Alto, CA: Consulting Psychologists Press.

Spitzer, R. L., Kroenke, K., Williams, J. B., and Lowe, B. (2006). A brief measure for assessing generalized anxiety disorder: the gad7. Arch. Int. Med. 166, 1092-1097. doi: 10.1001/archinte.166.10. 1092

Temsah, M. H., Al-Sohime, F., Alamro, N., Al-Eyadhy, A., Al-Hasan, K., Jamal, A., et al. (2020). The psychological impact of covid-19 pandemic on health care workers in a mers-cov endemic country. J. Infect. Public Health 13, 877-882. doi: 10.1016/j.jiph.2020.05.021

Tull, M. T., Edmonds, K. A., Scamaldo, K. M., Richmond, J. R., Rose, J. P., and Gratz, K. L. (2020). Psychological outcomes associated with stay-at-home orders and the perceived impact of covid-19 on daily life. Psychiatry Res. 289:113098. doi: 10.1016/j.psychres.2020.11 3098

Turkmen, D., Altunisik, N., Sener, S., and Colak, C. (2020). Evaluation of the effects of covid-19 pandemic on hair diseases through a webbased questionnaire. Dermatol. Ther. 33:e13923. doi: 10.1111/dth.1 3923

Wang, C., Pan, R., Wan, X., Tan, Y., Xu, L., Ho, C. S., et al. (2020). Immediate psychological responses and associated factors during the initial stage of the 2019 coronavirus disease (covid-19) epidemic among the general population in china. Int. J. Environ. Res. Public Health 17:1729. doi: 10.3390/ijerph1705 1729
Wang, M. W., Zhou, M. Y., Ji, G. H., Ye, L., Cheng, Y. R., Feng, Z. H., et al. (2020). Mask crisis during the covid-19 outbreak. Eur. Rev. Med. Pharmacol. Sci. 24, 3397-3399. doi: 10.26355/eurrev_202003_20707

Zhong, B. L., Luo, W., Li, H. M., Zhang, Q. Q., Liu, X. G., Li, W. T., et al. (2020). Knowledge, attitudes, and practices towards covid-19 among chinese residents during the rapid rise period of the covid-19 outbreak: a quick online cross-sectional survey. Int. J. Biol. Sci. 16, 1745-1752. doi: 10.7150/ijbs.45221

Conflict of Interest: The authors declare that the research was conducted in the absence of any commercial or financial relationships that could be construed as a potential conflict of interest.

Publisher's Note: All claims expressed in this article are solely those of the authors and do not necessarily represent those of their affiliated organizations, or those of the publisher, the editors and the reviewers. Any product that may be evaluated in this article, or claim that may be made by its manufacturer, is not guaranteed or endorsed by the publisher.

Copyright (c) 2021 Yang, Wu, Wang and Peng. This is an open-access article distributed under the terms of the Creative Commons Attribution License (CC BY).

The use, distribution or reproduction in other forums is permitted, provided the original author(s) and the copyright owner(s) are credited and that the original publication in this journal is cited, in accordance with accepted academic practice. No use, distribution or reproduction is permitted which does not comply with these terms. 